\title{
A CRITERION FOR DETERMINING THE SPACE OF IMMERSION OF A VARIETY OF ARBITRARY DIMENSIONALITY
}

\section{MARY DEAN CLEMENT}

1. Introduction. One of the classical developments in the field of projective differential geometry is a necessary and sufficient condition that a proper analytic curve be immersed in a linear space of given dimensionality. This condition is customarily expressed in. terms of an ordinary linear homogeneous differential equation, but may be expressed alternatively in terms of the rank of a certain matrix. The purpose of this paper is to extend the classical theory to obtain an analogous necessary and sufficient condition for the space of immersion of a proper analytic variety of arbitrary dimensionality. The criterion so obtained is expressed most concisely in terms of the rank of a certain matrix, but may be expressed in terms of a completely integrable system of linear homogeneous partial differential equations.

2. Definitions and hypotheses. In an $n$-dimensional projective space, $S_{n}$, let us consider a proper analytic variety, $V_{m}$, of $m$ dimensions $(m \leqq n)$. Let the variety be represented by the parametric vector equation $x=x\left(u_{1}, \cdots, u_{m}\right)$, where $x$ is the column vector whose $n+1$ components are the homogeneous projective coordinates of an ordinary point $P_{x}$ on the variety $V_{m}$. In order that the variety be analytic, we demand that these components be single-valued analytic functions of the $m$ independent parameters in a domain $D$ of the parameter space, such that a one-to-one continuous correspondence exist between points lying on the variety and sets of values of the parameters in $D$. The variety is proper if the coordinates of a variable point on it satisfy no linear partial differential equation of the first order. By the partial derivative of $x$ with respect to a parameter $u_{i}$ we shall mean the column vector each of whose components is obtained from the corresponding component of $x$ by differentiation with respect to $u_{i}$.

While the variety $V_{m}$ lies in the space $S_{n}$, it may be entirely contained in a linear subspace of fewer than $n$ dimensions. Let $S_{k}$ $(m \leqq k \leqq n)$ be called the space of immersion of $V_{m}$ if $V_{m}$ lies entirely in $S_{k}$ and does not lie in any subspace $S_{k-1}$.

Presented to the Society, April 27, 1951; received by the editors December 24, 1951. 
3. The set of matrices $M_{m}^{(h)}$. In this section we define a set of matrices associated with a given variety, and study some of the algebraic properties of these matrices which will enable us in $\$ 5$ to establish the desired geometric criterion.

Relative to a given variety $V_{m}$ at an ordinary point $P_{x}$, let us define a set of matrices $M_{m}^{(h)}(h \geqq 0)$ recursively: let $M_{m}^{(0)}$ be the column matrix (x); for $h>0$, let $M_{m}^{(h)}$ be formed from $M_{m}^{(h-1)}$ by adjoining as columns all the partial derivatives of $x$ of order $h$. Since the variety is taken to be analytic, partial derivatives of $x$ of all orders exist. Thus the matrix $M_{m}^{(h)}$ is defined for every non-negative integer $h$, and is unique except for the ordering of its columns.

From the definition it follows immediately that, for any $h$, the matrix $M_{m}^{(h)}$ is a submatrix of any $M_{m}^{(h+s)}(s \geqq 0)$; in fact, each column of $M_{m}^{(h)}$ is a complete column of $M_{m}^{(h+s)}$, since both matrices have the same number of rows, $n+1$. The number of columns of the matrix $M_{m}^{(h)}$ is clearly the same as the number of partial derivatives of $x$ of orders less than or equal to $h$ (including in this number the quantity $x$ considered as the zero-th partial derivative of itself); thus $M_{m}^{(h)}$ contains $C_{m+h, m}$ columns. The ranks of two particular matrices deserve especial attention: the matrix $M_{m}^{(0)}$ has rank 1, since not all the homogeneous coordinates of a point can be zero; the matrix $M_{m}^{(1)}$ has rank $m+1$, since this condition is equivalent to the condition that the variety $V_{m}$ be proper.

Another property of the set of matrices, fundamental for our purposes, is embodied in the following theorem.

THEOREM 1. If the matrices $M_{m}^{(h)}$ and $M_{m}^{(h+1)}$ have the same rank, $r$, then every matrix $M_{m}^{(h+s)}(s \geqq 0)$ has rank $r$.

Proof. The theorem is trivial for $s=0$ and $s=1$. The general case follows by a simple induction from the particular case $s=2$. We prove the theorem for $s=2$ by consideration of the differential equations which express linear dependence among the columns of the three matrices involved.

Since the matrix $M_{m}^{(h)}$ has rank $r$, it has $r$ linearly independent columns, say $A_{1}, \cdots, A_{r}$, such that any other column, say $B_{j}$, can be expressed as a linear combination of the $A_{i}$. That is, if

$$
M_{m}^{(h)}=\left(A_{i}, B_{j}\right) \quad\left(i=1, \cdots, r ; j=r+1, \cdots, C_{m+h, m}\right),
$$

then we may write the equations

$$
B_{i}=\sum_{i} b_{j i} A_{i}
$$


where the $b_{j i}$ are analytic scalar functions of the parameters $u_{1}, \cdots, u_{m}$, and the subscripts $i$ and $j$, here and throughout the proof of this theorem, have the ranges given in equation (1).

The matrix $M_{m}^{(n+1)}$ also contains the columns $A_{i}, B_{j}$. If we call the remaining columns $C_{p}$, we may write

$$
M_{m}^{(h+1)}=\left(A_{i}, B_{j}, C_{p}\right)
$$

with the subscript $p$, here and subsequently in this proof, taking the values $p=C_{m+h, m}+1, \cdots, C_{m+h+1, m}$. In this matrix the columns $A_{i}$ are still linearly independent, the columns $B_{j}$ still related to them by equations (2). Since by hypothesis the rank of $M_{m}^{(h+1)}$ is $r$, no column $C_{p}$ can be independent of the $A_{i}$; consequently we have the equations

$$
C_{p}=\sum_{i} c_{p i} A_{i}
$$

with the $c_{p i}$ analytic scalar functions of the parameters.

Let us now consider the matrix $M_{m}^{(n+2)}$. It contains all the columns $A_{i}, B_{j}, C_{p}$, and equations (2) and (3) hold. Let us call the remaining columns $D_{q}$, with $q=C_{m+h+1, m}+1, \cdots, C_{m+h+2, m}$. Any column $D_{q}$ is a derivative of $x$ of order $h+2$, and can be obtained, by a suitable differentiation of some $C_{p}$, as a linear combination of the $A_{i}$ and derivatives of the $A_{i}$. But any derivative of an $A_{i}$ is another $A_{i}$, a $B_{j}$, or a $C_{p}$. Hence from (2) and (3) any $D_{q}$ can be expressed as a linear combination of the $A_{i}$ alone. Thus we have the equations

$$
D_{q}=\sum_{i} d_{q i} A_{i}
$$

with the $d_{q i}$ analytic scalar functions of the parameters.

Equations (2), (3), and (4) assure us that only $r$ of the columns of the matrix $M_{m}^{(h+2)}$ are linearly independent, and thus the rank of $M_{m}^{(h+2)}$ is $r$.

CoRollaRy 1.1. If two matrices $M_{m}^{(h)}$ and $M_{m}^{(h+s)}$ have the same rank, $r$, then any matrix $M_{m}^{(n+t)}(0 \leqq t \leqq s)$ has rank $r$.

CoRollaRy 1.2. If the rank of $M_{m}^{(n+1)}$ exceeds the rank of $M_{m}^{(n)}$, then the rank of $M_{m}^{(h)}$ exceeds the rank of $M_{m}^{(h-1)}$.

The next two theorems and their corollaries, although they seem artificial in form, are of value in establishing the criterion toward which we are working. Their purpose is to determine certain conditions under which a matrix and a submatrix may have the same rank. 
TheoRem 2. If the matrix $M_{m}^{(h)}$ has rank $h+1$ then $M_{m}^{(h-m+1)}$ has rank $h+1$.

Proof. The theorem is trivial for $m=1$. For $m>1$, the theorem is proved by an indirect argument. For $m>1, M_{m}^{(n-m+1)}$ is a submatrix of $M_{m}^{(h)}$. Consequently, if we assume that $M_{m}^{(h-m+1)}$ has rank different from $h+1$, we must assume that it has rank at most $h$. Then by Corollary $1.2, M_{m}^{(h-m)}$ has rank at most $h-1$. Proceeding by induction, we find that the matrix $M_{m}^{(1)}$ has rank at most $m$. But this contradicts the fact, noted above, that $M_{m}^{(1)}$ has rank $m+1$. Thus our result is established.

CoROllaRY 2.1. For $m>1$, if the matrix $M_{m}^{(h)}$ has rank $h+1$, then $M_{m}^{(h-m+2)}$ has rank $h+1$.

This follows immediately from Theorem 2 and Corollary 1.1.

ThOeRem 3. If the matrix $M_{m}^{(h-m+2)}$ has rank $h+1$, then $M_{m}^{(h-m+1)}$ has rank $h+1$.

This theorem can be proved by an indirect argument similar to that of Theorem 2. The details of the proof will be omitted.

CoRollary 3.1. The matrix $M_{m}^{(h-m+2)}$ has rank $h+1$ if and only if $M_{m}^{(h-m+1+\lambda)}$ has rank $h+1$, for any positive integral value of $\lambda$.

This follows from Theorem 3 and Theorem 1 by an induction on $\lambda$.

4. The classical theorem. We turn now to a consideration of the geometric theorems which are the concern of this paper. In our proofs we shall employ the theorems and corollaries concerning matrices which were established in the preceding section.

The well known criterion for determining the space of immersion of a proper analytic curve may be phrased in terms of the rank of a matrix as follows:

THEOREM 4. A necessary and sufficient condition that a proper analytic curve $V_{1}: x=x(u)$ lying in a space $S_{n}$ be immersed in a subspace $S_{k}$ is that the $n+1$ by $k+2$ matrix

$$
M_{1}^{(k+1)}=\left(x, x^{\prime}, x^{\prime \prime}, \cdots, x^{(k+1)}\right)
$$

be of rank $k+1$.

Proof. Often this condition is given in terms of an ordinary linear homogeneous differential equation of order $k+1$, and is proved by means of the theory of differential equations $[1$, p. 5]. An alternative, geometric proof of the necessity of the condition will be presented 
here. If a curve $V_{1}$ is immersed in a space $S_{k}$, then at an ordinary point $P_{x}$ the osculating space $S_{k}$ of $V_{1}$ is determinate and is, in fact, the space of immersion, while the osculating $S_{k+1}$ of $V_{1}$ is indeterminate. This implies that the points with coordinates $x, x^{\prime}, \cdots$, $x^{(k)}$, which determine the osculating $S_{k}$ of $V_{1}$ at $P_{x}$, are linearly independent, while the points with coordinates $x, x^{\prime}, \cdots, x^{(k+1)}$ are linearly dependent. Thus the matrix $M_{1}^{(\boldsymbol{k})}$ is of rank $k+1$ and $M_{1}^{(\boldsymbol{k}+1)}$ is of rank $k+1$ also.

We shall defer proving the sufficiency of the condition, since it is easily obtained as a special case of the more general theorem to be studied in the next section.

5. The principal theorem. The criterion stated above for the space of immersion of a curve involves a matrix whose columns are $x$ and derivatives of $x$, defined at an ordinary point $P_{x}$ of the curve $V_{1}$. If we consider analogously a matrix with columns $x$ and partial derivatives of $x$, defined at an ordinary point $P_{x}$ of a variety $V_{m}$, and if we observe certain refinements which are justified by the theory of $\S 3$, we find that the generalization of $M_{1}^{(\boldsymbol{k}+1)}$ is $M_{m}^{(\boldsymbol{k}-m+2)}$, and we are led to the following theorem.

THEOREM 5. A necessary and sufficient condition that a proper analytic variety $V_{m}: x=x\left(u_{1}, \cdots, u_{m}\right)$ lying in a space $S_{n}$ be immersed in a subspace $S_{k}$ is that the $n+1$ by $C_{k+2, m}$ matrix $M_{m}^{(k-m+2)}$ be of rank $k+1$.

Proof. Two cases arise in the proof of the necessity of this condition, according as $m=1$ or as $m>1$. The first case has already been presented as a proof of the necessity of Theorem 4 .

For the case $m>1$, let us consider at an ordinary point $P_{x}$ of $V_{m}$ the space $S(h, 0)$, which may be thought of as a generalization of the osculating $S_{h}$ of a curve. The space $S(h, 0)$ is defined in the customary way $[2$, p. 307] as the linear space of least dimensions containing the osculating $S_{h}$ of all curves upon $V_{m}$ passing through $P_{x}$. The space $S(h, 0)$ is determined by the $C_{m+h, m}$ points whose coordinates make up the matrix $M_{m}^{(h)}$. For an unspecialized variety and for $n$ sufficiently large, these points are linearly independent and $S(h, 0)$ is of dimensionality $C_{m+h, m}-1$. Suppose, however, that $V_{m}$ is immersed in $S_{k}$. Then the space of immersion contains every curve upon $V_{m}$ which passes through $P_{x}$; it is the osculating $S_{k}$ of any curve upon $V_{m}$ which has a determinate osculating $S_{k}$ (that is, of any curve upon $V_{m}$ which is itself immersed in $S_{k}$ ); and thus by definition the $S(k, 0)$ of $V_{m}$ at $P_{x}$ coincides with the space of immersion. It follows at once that $S(k, 0)$ is of dimensionality $k$, and that only $k+1$ of the $C_{m+k, m}$ points defining $S(k, 0)$ are linearly independent. This implies 
that the matrix $M_{m}^{(\mathrm{k})}$ is of rank $k+1$. Corollary 2.1 now assures us that $M_{m}^{(k-m+2)}$ is of rank $k+1$.

The sufficiency of the condition may be proved by the following argument. If the matrix $M_{m}^{(k-m+2)}$ is of rank $k+1$, then by Theorem 3 and Corollary 3.1 every $M_{m}^{(h)}$ for $h \geqq k-m+1$ is of rank $k+1$. Thus every partial derivative of $x$, of any order whatsoever, can be expressed as a linear combination of $k+1$ linearly independent partial derivatives of orders at most $k-m+1$. Now if $P_{X}$ is any point on the variety $V_{m}$ sufficiently close to $P_{x}$, the coordinates $X$ may be expressed in a Taylor's expansion in terms of the coordinates $x$ and partial derivatives of $x$. But since all the partial derivatives of $x$ can be expressed linearly in terms of $k+1$ independent derivatives, we see that the point $P_{X}$ is linearly dependent upon $k+1$ points, and thus the variety $V_{m}$ is immersed in a space $S_{k}$.

From Theorem 5, by Theorem 3 and Corollary 3.1, we obtain the following:

COROLlaRy 5.1. If a variety $V_{m}$ is immersed in a space $S_{k}$, then the matrix $M_{m}^{(\mathrm{k}-m+1)}$ has rank $k+1$.

Corollary 5.2. A variety $V_{m}$ is immersed in a space $S_{k}$ if and only if the matrix $M_{m}^{(k-m+1+\lambda)}$ is of rank $k+1$, for any positive integral value of $\lambda$.

6. Concluding remarks. It is perhaps desirable to make the following comments upon Theorem 5 .

The theorem specializes easily for varieties of dimensionality from 1 to $k-1$. It is interesting to note that for $m=k$ the theorem indicates familiar conditions for linearity of a variety.

When we apply the foregoing theory to specific cases, we see that for many particular varieties the rank $k+1$ is achieved by some matrix $M_{m}^{(h)}$ for $h<k-m+1$. For example, consider the well known surface of Veronese, immersed in space $S_{5}$, which has parametric equations: $x_{1}=1, x_{2}=u, x_{3}=v, x_{4}=u^{2}, x_{6}=u v, x_{6}=v^{2}$. Straightforward computation shows that for this surface $M_{2}^{(2)}$ achieves the maximum rank, 6. In fact, a practical procedure for determining the space of immersion $S_{k}$ of a particular variety $V_{m}$ is to compute the ranks of successive matrices $M_{m}^{(0)}, M_{m}^{(1)}, \ldots$. When two successive matrices have equal ranks, then by Theorem 1 every subsequent matrix has the same rank, and this rank must be $k+1$. It is possible to show by counter examples, however, that there is no value $g$ less than $k-m+1$ such that $M_{m}^{(o)}$ achieves rank $k+1$ for all varieties. One such counter example is the tangent developable to the rational 
normal curve in space $S_{5}$. Parametric equations for this surface are: $x_{1}=1, \quad x_{2}=u+v, \quad x_{3}=u^{2}+2 u v, \quad x_{4}=u^{3}+3 u^{2} v, \quad x_{6}=u^{4}+4 u^{3} v, \quad x_{6}=u^{5}$ $+5 u^{4} v$. For this surface we find that $M_{2}^{(4)}$, which is $M_{2}^{(\mathbf{z}-m+1)}$, has rank 6 , but $M_{2}^{(h)}$ for $h<4$ has rank less than 6 .

The original criterion for the space of immersion of a curve has usually been expressed in terms of an ordinary linear homogeneous differential equation. The question naturally arises as to whether it is possible to express analogously our generalization of this theory. The affirmative answer to this question has already been implied, and a technique suggested, in the proofs of Theorems 1 and 5 . We recall that $M_{m}^{(k-m+2)}$ contains $C_{k+2, m}$ columns, of which only $k+1$ are linearly independent. Expressing the dependence of columns of this matrix in terms of differential equations, we have the following theorem.

TheOREM 6. $A$ necessary and sufficient condition that a proper analytic variety $V_{m}: x=x\left(u_{1}, \cdots, u_{m}\right)$ lying in a space $S_{n}$ be immersed in a subspace $S_{k}$ is that the coordinates $x$ of a variable point on $V_{m}$ satisfy a completely integrable system of $C_{k+2, m}-(k+1)$ linearly independent linear homogeneous partial differential equations of orders $k-m+2$ or less.

In a similar fashion, Corollaries 5.1 and 5.2 can be interpreted to yield the following:

CoROllaRy 6.1. If a variety $V_{m}$ is immersed in a space $S_{k}$, then the coordinates $x$ of a variable point on $V_{m}$ satisfy $C_{k+1, m-1}$ linearly independent linear homogeneous partial differential equations of order $k-m+2$.

Corollary 6.2. A variety $V_{m}$ is immersed in a space $S_{k}$ if and only if the coordinates of a variable point on $V_{m}$ satisfy a completely integrable system of $C_{k+1+\lambda, m}-(k+1)$ linearly independent linear homogeneous partial differential equations of orders $k-m+1+\lambda$ or less, for any positive integral value of $\lambda$.

It should be borne in mind that while these partial differential equations form a completely integrable system, and while they are linearly independent, at the same time they are not necessarily analytically independent, in the sense that many of the equations of the system can be obtained from other equations of the system by differentiation and linear combination. The determination of the smallest possible number of these differential equations which are analytically independent as well as linearly independent is a problem which the author expects to consider in a future paper. 


\section{REFERENCES}

1. E. P. Lane, $A$ treatise on projective differential geometry, Chicago, 1942.

2. E. Bompiani, Alcune proprietd proiettivo-differenziali dei sistemi di rette negli iperspazi, Rend. Circ. Mat. Palermo vol. 37 (1914) pp. 305-331.

The University of Chicago

\section{THE RECIPROCAL OF A CONTINUED FRACTION}

\section{W. T. SCOTT}

Stieltjes [3, Chapter X], ${ }^{1}$ and later Rogers [2], gave formulas by means of which the reciprocal continued fractions for continued fractions of a certain class may be determined. We give below a theorem which extends the class of continued fractions to which this reciprocal transformation is applicable ${ }^{2}$ moreover, the theorem is stated in terms of certain parameters which facilitate the transformation.

From results of [1] it is known that there is a unique, one-to-one correspondence between formal power series, $F(z)=1+\sum_{1}^{\infty} c_{p} / z^{p}$, and $C$-fractions,

$$
F(z) \sim 1+\frac{a_{1}}{z^{\beta_{1}}}+\frac{a_{2}}{z^{\beta_{2}}}+\cdots+\frac{a_{n}}{z^{\beta_{n}}}+\cdots,
$$

where the $\beta_{p}$ are integers, $\beta_{0}=0, \beta_{p-1}+\beta_{p}>0$, and $a_{p} \neq 0, p=1,2, \cdots$, $n$, or $p=1,2, \cdots$, according as the $C$-fraction does or does not terminate. The power series for the $p$ th approximant of the $C$-fraction agrees with $F(z)$ for the first $\left(\sigma_{p-1}+\sigma_{p}\right)$ terms, where $\sigma_{p}=\beta_{0}$ $+\beta_{1}+\cdots+\beta_{p}$; this property characterizes the correspondence (1).

For simplicity we put $a_{2 n}=0$ in case a $C$-fraction terminates with the $(2 n-1)$ th partial quotient. Our result is stated below for a class of terminating $C$-fractions. In the nonterminating case the conditions of the theorem must. hold for every index, $n$; consequently, a statement of our result in this case is obtained by replacing $n$ by $\infty$.

Presented to the Society, November 25, 1949; received by the editors February 1, 1952.

${ }^{1}$ Numbers in brackets refer to the bibliography at the end of the paper.

2 Another theorem on reciprocals of $C$-fractions was given by Evelyn Frank, Amer. J. Math. vol. 68 (1946) pp. 89-108. The domain of application of the two theorems does not overlap. 